\title{
Delayed functional independence after thrombectomy: temporal characteristics and predictors
}

\author{
Shashvat M Desai, ${ }^{1}$ Daniel A Tonetti, ${ }^{2}$ Andrew A Morrison, ${ }^{3}$ Bradley J Molyneaux, ${ }^{4}$ \\ Matthew Starr, ${ }^{4}$ Marcelo Rocha (D) , ${ }^{4}$ Bradley A. Gross, ${ }^{2}$ Brian Jankowitz, ${ }^{5}$ \\ Tudor G Jovin, ${ }^{6}$ Ashutosh P Jadhav (iD ${ }^{4}$
}

${ }^{1}$ Department of Neurology and Neurosurgery, University of Pittsburgh, Pittsburgh,

Pennsylvania, USA

${ }^{2}$ Department of Neurosurgery, University of Pittsburgh, Pittsburgh, Pennsylvania, USA ${ }^{3}$ University of Pittsburgh School of Medicine, Pittsburgh, Pennsylvania, USA

${ }^{4}$ Department of Neurology, University of Pittsburgh, Pittsburgh, Pennsylvania, USA ${ }^{5}$ Cooper Neurological Institute, Cooper University Hospital Camden, New Jersey, USA ${ }^{6}$ Department of Neurology, Cooper University Hospital, Camden, New Jersey, USA

Correspondence to Dr Ashutosh P Jadhav, Department of Neurology, University of Pittsburgh, Pittsburgh, PA 15213-2582, USA; jadhav.library@gmail.com

Received 2 April 2020 Revised 5 May 2020 Accepted 7 May 2020 Published Online First 2 June 2020

Check for updates

(C) Author(s) (or their employer(s)) 2020. No commercial re-use. See rights and permissions. Published by BMJ.

To cite: Desai SM,

Tonetti DA, Morrison AA

et al. J Neurolntervent Surg

2020:12:837-841.

\section{ABSTRACT \\ Background Variability in early neurological} improvement after endovascular thrombectomy (EVT) for large vessel occlusion (LVO) stroke is well documented. Understanding the temporal progression of functional independence after EVT, especially delayed functional independence in patients who do not experience early improvement, is essential for prognostication and rehabilitation.

Objective To determine the incidence of early and delayed functional independence and identify associated predictors after EVT.

Methods A retrospective analysis of prospectively collected data on patients undergoing EVT in the setting of anterior circulation LVO was performed. Demographic, clinical, radiological, treatment, and procedural information were analyzed. Incidence and predictors of early functional independence (EFI, modified Rankin Scale (mRS) score $0-2$ at discharge) and delayed functional independence (DFI, mRS score $0-2$ at 90 days in non-EFI patients) were analyzed.

Results Three hundred and fifty-five patients met the study criteria. 55\% were women and mean age was $71 \pm 15$. Mean National Institutes of Health Stroke Scale (NIHSS) score was $17 \pm 6$ and median Alberta Stroke Program Early CT Score was 9 (8-10). EFI was observed in $21 \%$ (73) of patients. Among non-EFI patients (282), DFI was observed in 30\% (85) of patients. Shorter time to treatment $(p=0.03)$, lower 24 hours NIHSS score $(p<0.001)$, and smaller follow-up infarct volume $(p=0.003)$ were independent predictors of EFI. Younger age $(p=0.011)$, lower 24 hours NIHSS score $(p=0.001)$, and absence of parenchymal hemorrhage ( $\mathrm{PH} 2$; $p=0.039$ ) were independent predictors of DFI. Conclusion Approximately one-fifth of patients experience $\mathrm{EFI}$ and one-third of non-early improvers experience DFI. Younger age, lower 24 hours NIHSS score, and absence of parenchymal hemorrhage were independent predictors of DFI among non-early improvers. Further studies are required to improve our understanding of DFI.

\section{INTRODUCTION}

Endovascular thrombectomy (EVT) is the standard of care for anterior circulation large vessel occlusion (LVO) strokes. ${ }^{1}$ Despite strict patient selection and high rates of recanalization with
EVT in randomized controlled trials, only $\sim 50 \%$ of patients achieve functional independence at 90 days. ${ }^{2}$ Heterogeneity in early neurological improvement after EVT is well-documented. At 24 hours following EVT, approximately $20 \%$ of patients have a National Institutes of Health Stroke Scale (NIHSS) score of $0-2 .^{2-4}$ Furthermore, delayed functional independence is observed in a subset of patients who do not experience early improvement. This phenomenon has been referred to as 'stunned brain'. ${ }^{56}$ Understanding the temporal progression of functional independence after EVT is essential for prognostication and rehabilitation. Recanalization dynamically alters stroke injury and recovery mechanisms and represents a key initial step to improve outcomes after LVO stroke. Data on characteristics and predictors of early (EFI) and delayed functional improvements (DFI) after EVT are limited. In this study, we aim to describe the characteristics and incidence of EFI and DFI and identify associated predictors after EVT.

\section{METHODS}

Using the Get With the Guidelines database, a retrospective analysis of prospectively collected data was performed for all patients presenting with acute ischemic stroke to a comprehensive stroke center between January 2015 and February 2018. Demographic characteristics, clinical and radiological data, and treatment and procedural information were collected and analyzed. This study was approved by the local institutional review board. Data used to prepare this manuscript may be made available on reasonable request.

\section{Patient selection}

Patients with acute ischemic stroke who presented to a single comprehensive stroke center and underwent EVT during the study period were analyzed. Occlusion location was confirmed by CT angiography, magnetic resonance angiography, and/or cerebral angiography. The decision to offer endovascular therapy was determined by a vascular neurologist and treating neurointerventionalist after detailed discussion with the patient and their family. Patients with good baseline functional status (modified Rankin Scale (mRS) score 0-2) who received EVT for occlusion of an anterior circulation artery 


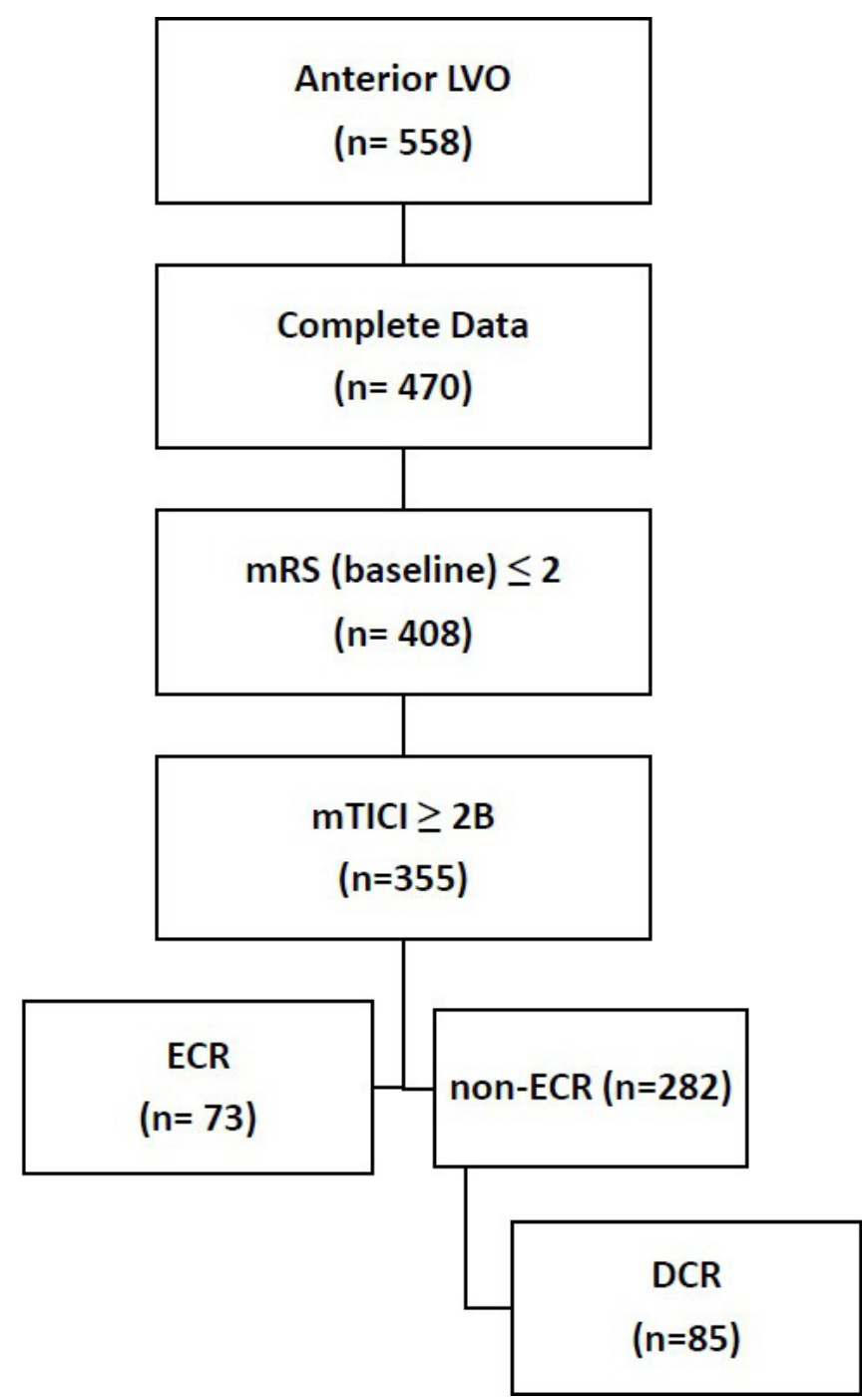

Figure 1 Flow chart. DFI, delayed functional independence; EFI, early functional independence; LVO, large vessel occlusion; mRS, modified Rankin Scale; mTICl, modified Thrombolysis in Cerebral Infarction.

(intracranial internal carotid artery and/or middle cerebral artery segment 1 (M1)) and achieved successful recanalization (modified Thrombolysis in Cerebral Infarction (mTICI) $\geq 2 b$ ) were included in the study.

\section{Baseline characteristics}

Baseline demographic (age, sex), clinical (stroke severity, time from symptom onset, risk factor profile), and radiographic (pretreatment Alberta Stroke Program Early CT Score (ASPECTS), occlusion location) data, and information on procedural technique and efficiency were collected and analyzed by a vascular neurologist blinded to patient outcomes. Additionally, follow-up infarct volumes (FIVs) were manually calculated (product of slice thickness and sum of area of region of interest across all slices) on the postprocedure ( $24 \pm 12$ hours) CT head or MRI-DWI.

\section{Outcomes}

Early functional independence (EFI) was defined as mRS score $0-2$ at discharge. Consequently, delayed functional independence was defined as achieving mRS score 0-2 at 90 days among non-EFI patients. Functional independence was determined by
mRS score in the clinic setting by a vascular neurologist $(65 \%)$ or via telephone by a specialized nursing professional trained in stroke care $(35 \%)$. Safety outcomes included parenchymal hemorrhage (PH1 or $\mathrm{PH} 2)$, symptomatic intracranial hemorrhage, and mortality.

\section{Statistical analyses}

Continuous variables are reported as mean \pm SD or median with interquartile range (as appropriate), and categorical variables are reported as proportions. Between-groups comparison for continuous variables was performed using Student's t-test and categorical variables using Chi-square test or Fisher exact test, as appropriate. Univariable analysis was performed for baseline characteristics between EFI and non-EFI groups as well as between DFI and non-DFI subgroups (among non-EFI patients). Exploratory analyses were performed, comparing EFI with DFI groups. Multivariable logistic regression analysis was performed to identify predictors of DFI. Variables with a $\mathrm{p}$ value of $<0.2$ on univariate analysis were used for multivariable logistic regression. Our analysis was performed on patients achieving mTICI $\geq 2 \mathrm{~b}$ recanalization. Associations are presented as OR with $95 \%$ CI. Significance was defined as $\mathrm{p}$ value $\leq 0.05$. Statistical analysis was performed using IBM SPSS Statistics 23 (IBMArmonk, New York, USA).

\section{RESULTS}

\section{Patient demographics}

Three hundred and fifty-five patients met the study criteria (figure 1). Fifty-five percent of patients were female and mean age was $71 \pm 15$ years. Mean NIHSS score was $17 \pm 6$ and median ASPECTS was 9 (IQR 8-10). Mean time last known well (TLKW) to comprehensive stroke center arrival was $9 \pm 11.6$ hours and 59\% (211) of patients received EVT within 6 hours. IV tissue plasminogen activator (tPA) was received by 36\% (128), and $34 \%$ (121) harbored an internal carotid artery occlusion. Highly successful recanalization ( $\mathrm{mTICI} \geq 2 \mathrm{c}$ ) was achieved in $15 \%(54)$ of patients. Overall, mRS score 0-2 at 90 days was observed in

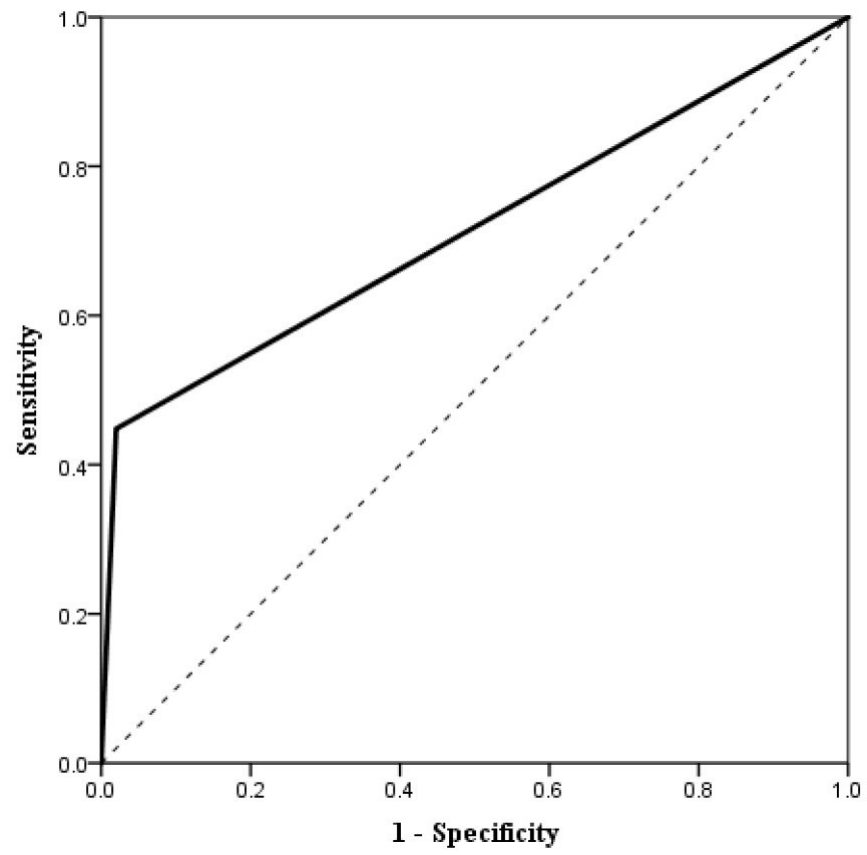

Figure 2 Receiver operating curve for the ability of early functional independence to predict modified Rankin Scale score $0-2$ at 90 days. 
Table 1 Baseline characteristics and outcomes

\begin{tabular}{|c|c|c|c|c|c|c|}
\hline \multirow[b]{2}{*}{ Characteristics and outcomes } & \multirow[b]{2}{*}{$\operatorname{EFI}(73)$} & \multirow[b]{2}{*}{ Non-EFI (282) } & \multirow[b]{2}{*}{$P$ value } & \multicolumn{3}{|c|}{ Among non-EFI (282) } \\
\hline & & & & DFI (85) & Non-DFI (197) & $P$ value \\
\hline Mean age (SD) & $65.3(14.3)$ & $71.9(14.6)$ & 0.001 & $67.0(15.4)$ & $74.0(13.8)$ & 0.000 \\
\hline Male sex, \% (n) & $50.7(37)$ & $43.6(123)$ & 0.294 & $48.2(41)$ & $41.6(82)$ & 0.360 \\
\hline Mean pretreatment NIHSS score (SD) & $13.2(5.7)$ & $17.8(5.8)$ & 0.000 & $16.9(6.4)$ & $18.1(5.5)$ & 0.103 \\
\hline Median ASPECTS (IQR) & $9(9-10)$ & $9(8-10)$ & 0.018 & $9(9-10)$ & $9(8-10)$ & 0.124 \\
\hline Mean TLKW to arrival (SD) & $398.2(382.5)$ & $582.8(755.9)$ & 0.049 & $544.1(669.6)$ & $599.4(791.1)$ & 0.578 \\
\hline TLKW to arrival $>6$ hours, $\%$ (n) & $32.9(24)$ & $42.6(120)$ & 0.143 & $37.6(32)$ & $44.7(88)$ & 0.296 \\
\hline Hypertension, \% (n) & $63.0(46)$ & $77.3(218)$ & 0.016 & $67.1(57)$ & $81.7(161)$ & 0.009 \\
\hline Hyperlipidemia, \% (n) & $46.6(34)$ & $55.3(156)$ & 0.190 & $44.7(38)$ & $59.9(118)$ & 0.020 \\
\hline Diabetes mellitus, \% (n) & $13.7(10)$ & $25.5(72)$ & 0.042 & $20.0(17)$ & $27.9(55)$ & 0.182 \\
\hline Atrial fibrillation, \% (n) & $28.8(21)$ & $37.6(106)$ & 0.172 & $29.4(25)$ & $41.1(81)$ & 0.062 \\
\hline Smoking, \% (n) & $32.9(24)$ & $23.0(65)$ & 0.096 & $31.8(27)$ & $19.3(38)$ & 0.030 \\
\hline Prior stroke, \% (n) & $17.8(13)$ & $17.7(50)$ & 1.000 & $12.9(11)$ & $19.8(39)$ & 0.179 \\
\hline CAD/CHF \% (n) & $28.8(21)$ & $29.4(83)$ & 1.000 & $22.4(19)$ & $32.5(64)$ & 0.090 \\
\hline Occlusion location: internal carotid artery, \% (n) & $28.8(21)$ & $35.5(100)$ & 0.333 & $32.9(28)$ & $36.5(72)$ & 0.590 \\
\hline IV tPA, \% (n) & $41.1(30)$ & $34.8(98)$ & 0.340 & $45.9(39)$ & $29.9(59)$ & 0.014 \\
\hline $\mathrm{mTICl} \geq 2 \mathrm{c}, \%(\mathrm{n})$ & $23.3(17)$ & $13.1(37)$ & 0.043 & $14.1(12)$ & $12.7(25)$ & 0.848 \\
\hline Mean 24 hours NIHSS score (SD) & $2.9(3.5)$ & $12(7.9)$ & $<0.001$ & $8.1(4.9)$ & $13.2(8.4)$ & $<0.001$ \\
\hline $\mathrm{PH} 1 / 2, \%(\mathrm{n})$ & $6.8(5)$ & $24.5(69)$ & 0.001 & $15.3(13)$ & $28.4(56)$ & 0.023 \\
\hline Follow-up infarct volume $(\mathrm{mL})$ & $10.2 \pm 11.2$ & $61.9 \pm 71.9$ & $<0.001$ & $37.2 \pm 39.4$ & $72.2 \pm 79.6$ & $<0.001$ \\
\hline $\mathrm{mRS}$ score $0-2$ at 90 days, $\%$ (n) & $94.5(69)$ & $30.1(85)$ & 0.000 & $100(85)$ & Mean mRS $4.6 \pm 1.2$ & NA \\
\hline Mortality, \% (n) & $4.1(3)$ & $26.6(75)$ & 0.000 & $0(0)$ & $38.1(75)$ & NA \\
\hline
\end{tabular}

ASPECTS, Alberta Stroke Program Early CT Score; CAD, coronary artery disease; CHF, congestive heart failure; DFI, delayed functional independence; $\mathrm{EFI}$, early functional independence; mRS, modified Rankin Scale; mTICl, modified Thrombolysis in Cerebral Infarction; NIHSS, National Institutes of Health Stroke Scale; PH, parenchymal hemorrhage; TLKW, time last known well; tPA, tissue plasminogen activator.

$43.4 \%$ (154), mortality at 90 days occurred in $22 \%(78)$, and mean FIV was $51.8 \pm 67.8 \mathrm{~mL}$.

\section{Early and delayed functional independence}

Modified Rankin Scale score 0-2 at discharge (EFI) was observed in $21 \%(n=73)$ of patients and the remainder $(79 \%)$ did not experience EFI (non-EFI). Of the 282 patients who did not experience EFI, 85 (30\%) eventually achieved DFI (figure 1).

\section{Predictive value of EFI}

The proportion of patients with mRS score 0-2 at 90 days was significantly higher in the EFI group than in the non-EFI group (95\% vs $30 \%, \mathrm{p} \leq 0.01)$. The positive and negative predictive values of EFI to achieve mRS score 0-2 at 90 days were $94.5 \%$ and $69.8 \%$, respectively. The receiver operator curve analysis for the ability of EFI to predict mRS score 0-2 at 90 days had an area under the curve of $0.71(0.65-0.77, \mathrm{p} \leq 0.01)$ (figure 2 ). Additionally, $55.2 \%$ of patients with mRS score $0-2$ at 90 days had not experienced EFI.

\section{Early functional independence versus non-early functional independence}

In univariate analysis comparing patients with EFI with non-EFI, those who experienced EFI were significantly younger (65 vs 72 years, $\mathrm{p}=0.001)$ and had lower rates of hypertension $(63 \%$ vs $77 \%, \mathrm{p}=0.016)$ and diabetes mellitus $(14 \%$ vs $26 \%, \mathrm{p}=0.042)$. EFI patients had significantly lower NIHSS score at presentation $(13 \%$ vs $18 \%, \mathrm{p}<0.01)$ and a shorter time from last known well to arrival at a comprehensive stroke center (6.6 vs 9.7 hours, $\mathrm{p}=0.049$ ). Patients with EFI had higher rates of mTICI $\geq 2 \mathrm{c}$ (23\% vs $13 \%, \mathrm{p}=0.043)$ and experienced lower incidence of $\mathrm{PH} 1 / 2(7 \%$ vs $25 \%, \mathrm{p}<0.01)$. FIV was significantly lower in EFI patients $(10.2 \pm 11.2$ vs $61.9 \pm 71.9 \mathrm{~mL}, \mathrm{p} \leq 0.001)$. Finally, EFI patients demonstrated significantly higher rates of $\mathrm{mRS}$ score $0-2$ at 90 days $(95 \%$ vs $30 \%, \mathrm{p}<0.01)$ and lower all-cause mortality at 90 days $(4 \%$ vs $27 \%, \mathrm{p}<0.001)$. In multivariate analysis, shorter TLKW to arrival $(\mathrm{OR}=0.99(0.98-1), \mathrm{p}=0.03)$, lower 24 hours NIHSS score $(\mathrm{OR}=0.76(0.67-0.85), \mathrm{p}<0.001)$ and FIV $(\mathrm{OR}=0.96(0.93-0.98), \mathrm{p}=0.003)$ were independent predictors of EFI (table 1).

\section{Delayed functional independence versus non-delayed functional independence}

In univariate analysis comparing patients with DFI with nonDFI, patients who experienced DFI were significantly younger (67 vs 74 years, $p<0.01$ ) and had a significantly lower proportion of vascular risk factors, including hypertension $(67 \%$ vs $82 \%, p=0.009)$, hyperlipidemia ( $45 \%$ vs $60 \%, p=0.02)$, and tobacco use $(32 \%$ vs $19 \%, \mathrm{p}=0.03)$. They had a higher proportion of IV tPA use $(46 \%$ vs $30 \%, p=0.014)$, reduced occurrence of PH1/2 (15\% vs $28 \%, \mathrm{p}=0.023)$, and lower FIV $(37.2 \pm 39.4$ vs $72.2 \pm 79.6, \mathrm{p} \leq 0.001)$. In multivariate analysis, younger age $(p=0.011)$, lower 24 hours NIHSS score $(p=0.001)$ and absence of parenchymal hemorrhage $(\mathrm{PH} 2 ; \mathrm{p}=0.039)$ were independent predictors of DFI. Rates of EFI ( $22 \%$ vs $16 \%, \mathrm{p}=0.18)$ and DFI (31\% vs 27\%, p=0.47) were comparable between American Heart Association (AHA)/American Stroke Association eligible and non-eligible patients (table 1). 
Table 2 EFI, DFI and mRS score 0-2 predictor

\begin{tabular}{llll}
\hline & EFI predictors & DFI predictors & $\begin{array}{l}\text { Overall, mRS 0-2 } \\
\text { predictors }\end{array}$ \\
\hline $\begin{array}{l}\text { Significant } \\
\text { predictors on }\end{array}$ & Lower 24hours & Lower 24 hours & Lower 24 hours \\
multivariate & NIHSS & NIHSS & NIHSS \\
analyses & Lower FIV & Younger age & Younger age \\
& Shorter time to & Absence of PH2 & $\begin{array}{l}\text { Lower FIV } \\
\text { treatment }\end{array}$ \\
& & & $\begin{array}{l}\text { Higher ASPECTS } \\
\text { Absence of PH2 }\end{array}$ \\
\hline
\end{tabular}

ASPECTS, Alberta Stroke Program Early CT Score; DFI, delayed functional independence; EFI, early functional independence; FIV, follow-up infarct volume; mRS, modified Rankin Scale; NIHSS, National Institutes of Health Stroke Scale; PH, parenchymal hemorrhage.

Predictors of EFI, DFI, and mRS score 0-2 at 90 days are shown in table 2.

\section{Early functional independence versus delayed functional independence}

In univariate analysis comparing patients with EFI with those with DFI, EFI and DFI patients were comparable. Regression analyses identified shorter time to treatment $(p=0.047)$ and lower FIV $(\mathrm{p}=0.001)$ as being associated with EFI in comparison with DFI (table 3$)$.

\section{DISCUSSION}

The main finding of our study is that among patients with anterior circulation LVO strokes who received EVT, 21\% experience

\begin{tabular}{|c|c|c|c|}
\hline & $\operatorname{EFI}(n=73)$ & DFI $(n=85)$ & $P$ value \\
\hline Mean age (SD) & $65.3(14.3)$ & $67.0(15.4)$ & 0.492 \\
\hline Male sex, \% (n) & $50.7(37)$ & $48.2(41)$ & 0.873 \\
\hline Mean NIHSS score (SD) & $13.2(5.7)$ & $16.9(6.3)$ & 0.191 \\
\hline Median ASPECTS (IQR) & $9(9-10)$ & $9(9-10)$ & 0.219 \\
\hline $\begin{array}{l}\text { Mean TLKW to arrival } \\
\text { (SD) }\end{array}$ & $398.2(382.5)$ & $544.1(669.6)$ & 0.109 \\
\hline $\begin{array}{l}\text { TLKW to arrival } \\
>6 \text { hours, \% (n) }\end{array}$ & $32.9(24)$ & $37.6(32)$ & 0.617 \\
\hline Hypertension, \% (n) & $63.0(46)$ & $67.1(57)$ & 0.619 \\
\hline Hyperlipidemia, \% (n) & $46.6(34)$ & $44.7(38)$ & 0.873 \\
\hline Diabetes mellitus, \% (n) & $13.7(10)$ & $20.0(17)$ & 0.397 \\
\hline Atrial fibrillation, \% (n) & $28.8(21)$ & $29.4(25)$ & 1.000 \\
\hline Smoking, \% (n) & $32.9(24)$ & $31.8(27)$ & 1.000 \\
\hline Prior stroke, \% (n) & $17.8(13)$ & $12.9(11)$ & 0.506 \\
\hline $\mathrm{CAD} / \mathrm{CHF}_{1} \%(\mathrm{n})$ & $28.8(21)$ & $22.4(19)$ & 0.366 \\
\hline $\begin{array}{l}\text { Occlusion location: } \\
\text { internal carotid artery, } \\
\% \text { (n) }\end{array}$ & $28.8(21)$ & $32.9(28)$ & 0.608 \\
\hline IV tPA, \% (n) & $41.1(30)$ & $45.9(39)$ & 0.630 \\
\hline $\mathrm{mTICl} \geq 2 \mathrm{c}, \%(\mathrm{n})$ & $23.3(17)$ & $14.1(12)$ & 0.154 \\
\hline $\begin{array}{l}\text { Follow-up infarct volume } \\
(\mathrm{mL})\end{array}$ & $10.2 \pm 11.2$ & $37.2 \pm 39.4$ & $<0.001$ \\
\hline $\mathrm{PH} 1 / 2, \%(\mathrm{n})$ & $6.8(5)$ & $15.3(13)$ & 0.132 \\
\hline
\end{tabular}

ASPECTS, Alberta Stroke Program Early CT Score; CAD, coronary artery disease; CHF, congestive heart failure; $\mathrm{DFI}$, delayed functional independence; $\mathrm{EFI}$, early functional independence; $\mathrm{mTICl}$, modified Thrombolysis in Cerebral Infarction; NIHSS, National Institutes of Health Stroke Scale; PH, parenchymal hemorrhage; TKLW, time last known well; tPA, tissue plasminogen activator. early functional independence and $30 \%$ of non-early improvers experience delayed functional independence. Predictors of early and delayed functional independence are distinct. Predictors of early functional independence include shorter time to treatment, lower 24 hours NIHSS score, and smaller follow-up infarct volume, whereas predictors of delayed functional independence after no early improvement include younger age, lower 24 hours NIHSS score, and absence of parenchymal hemorrhage.

Post-EVT improvement in stroke severity ${ }^{7-9}$ and NIHSS scorebased trajectory groups (patients experiencing largest improvement in NIHSS score within 2 days after thrombectomy $)^{10}$ have high sensitivity and specificity for predicting long-term outcome. Our study confirms this previous observation and finds that EFI can positively predict 90-day functional independence (positive predictive value 95\%). However, we also find that the lack of EFI cannot rule out eventual functional independence, as DFI was observed in $30 \%$ of non-early improvers. Furthermore, of all patients with $\mathrm{mRS}$ score $0-2$ at 90 days, $55 \%$ did not have mRS 0-2 at hospital discharge.

Similar to our study, prior reports have identified younger age, lower NIHSS score, and higher ASPECTS as predictors of functional independence at 90 days after EVT. ${ }^{2}$ A subset of patients who do not experience early improvement will experience DFI. Prior studies have not reported on the incidence and predictors of DFI.

In the absence of early clinical improvement, it is important to guide care and manage expectations. Our large retrospective series adds important insight into the real-world incidence of DFI and its predictors. The frequency of DFI in this study is consistent with prior studies. Bang et $a l^{6}$ found that among the 69 patients who received IV tPA or EVT, $40 \%$ experienced functional independence in a delayed fashion. Alexandrov and colleagues analyzed middle cerebral artery M1 and M2 strokes receiving IV tPA and reported that approximately 37\% patients with recanalization experienced ischemic stunning and eventually had delayed recovery. ${ }^{5}$

Potential reasons for lack of early improvement despite adequate recanalization include vascular factors (proximal re-occlusion of the index lesion, ${ }^{11} 12$ persistent or new distal occlusion in a small caliber vessel ${ }^{13}$ supplying eloquent parenchyma), microcirculation failure (no-reflow phenomenon), ${ }^{14} 15$ and parenchymal factors (large volume of infarcted parenchyma, futile reperfusion into little salvageable penumbra or dead brain tissue, or ischemic-reperfusion injury). ${ }^{16}{ }^{17}$ Parenchymal edema due to ischemia, hemorrhage in ischemic bed, ${ }^{18}$ and/or yet to be delineated injury mechanisms due to persistent ischemic environment may also play a role. Resolution of edema ${ }^{19}$ resolution of hemorrhage, delayed recovery of cerebral microcirculation, and increase in stroke healing mechanisms (plasticity of cortical projections ${ }^{20}$ reorganization of damaged neurons, ${ }^{21}$ and contralateral hemispheric compensation) over time may contribute to delayed functional independence after EVT.

Stunned brain may be mechanistically comparable to stunned heart. Stunned myocardium is viable myocardium salvaged by coronary reperfusion that exhibits prolonged post-ischemic dysfunction after reperfusion. ${ }^{22}$ Predictors of late improvement in contractile function after acute myocardial function include early treatment (associated with smaller infarct burden) and higher contractile reserve. ${ }^{23}$ In the brain, these determinants may be comparable to pretreatment NIHSS score and age, respectively.

In this study, we have established a temporal change in the predictors of good outcome. Predictors of EFI in our study population include shorter time to treatment, lower 24 hours 
NIHSS score, and lower FIV, indicating the importance of stroke severity, stroke burden and time. In the delayed phase, predictors of good outcome include younger age, lower 24 hours NIHSS score, and absence of hemorrhage. These findings suggest that recovery mechanisms are more robust in the young, are related to stroke severity after treatment, and are more effective in the absence of irritants like blood.

DFI represents an important subgroup of patients with respect to prognostication and rehabilitation resource allocation after EVT. Prevention of parenchymal hemorrhage, even when silent, may be an opportunity to improve outcomes. The combined finding of the predictive value of EFI and factors predicting DFI among non-early improvers may be used to refine efficacy end points in future randomized controlled trials exploring the benefit of neuroprotection (in the acute phase), stems cells (in the acute and subacute phases), and stroke rehabilitation interventions (delayed phase).

\section{Strengths}

Our study has several strengths. Data on DFI in the era of EVT are scant. We included patients with good baseline status and successful recanalization to create a homogeneous dataset. Additionally, we incorporated known and strong predictors of outcomes in our regression analyses, including 24 hours NIHSS score and FIV. Approximately $30 \%$ of our patients do not meet current AHA guidelines for EVT and reflect real-world practice. Our rates of DFI are consistent with previous reports from patients treated with IV-tPA.

\section{Limitations}

Our study is limited by its retrospective nature. Furthermore, our data limit us from studying more granular temporal trends in DFI between discharge and 90 days. Observation beyond 90 days may increase the number of patients experiencing DFI. Finally, our study focuses on clinical outcome as measured by the categorical scale of mRS. A more detailed analysis of recovery itself will require a more comprehensive assessment of functionality at baseline and follow-up, using for example the FIM or ARAT score.

\section{CONCLUSION}

Early functional independence is frequent and occurs in about one-third of patients with stroke who do no experience early functional independence after EVT. Younger age, lower 24 hours NIHSS score, and absence of parenchymal hemorrhage predict delayed functional independence among non-EFI patients. Further studies are required to confirm our findings and improve our understanding of delayed functional independence.

Twitter Shashvat M Desai @shashvatdesaiMD and Ashutosh P Jadhav @ ashupjadhav

Contributors Conception and design: SMD, APJ; acquisition of data: SMD, DAT, AAM; analysis and interpretation of data: all authors; drafting the article: SMD, APJ; critically revising the article: all authors; administrative/technical/material support: all authors; study supervision: APJ.

Funding Research reported in this publication was supported by the National Institute of Neurological Disorders and Stroke of the National Institutes of Health under Award Number U10NS086489 (NIH Stroke Trials Network-Regional Coordinating Center Univ. of Pittsburgh).

Competing interests BAG: consultant: Microvention; BJ: consultant: Medtronic; TGJ: consultant: Stryker Neurovascular (PI DAWN-unpaid), ownership interest:
Anaconda, advisory board/investor; Free0x Biotech, advisory board/investor; Route92, advisory board/investor; Blockade Medical, consultant; honoraria: Cerenovus.

\section{Patient consent for publication Not required.}

Ethics approval The study obtained ethics approval from the institutional review board (IRB) of the University of Pittsburgh [STUDY20010015] and we did not require informed consent from patients because the IRB approved 'waiver of consent' given the observational retrospective nature of the study.

Provenance and peer review Not commissioned; externally peer reviewed.

\section{ORCID iDs}

Marcelo Rocha http://orcid.org/0000-0002-4503-5827

Ashutosh P Jadhav http://orcid.org/0000-0002-9454-0678

\section{REFERENCES}

1 Powers WJ, Rabinstein AA, Ackerson T, et al. 2018 guidelines for the early management of patients with acute ischemic stroke: a guideline for healthcare professionals from the American Heart Association/American Stroke Association. Stroke 2018;49:e46-110.

2 Goyal M, Menon BK, van Zwam WH, et al. Endovascular thrombectomy after large-vessel ischaemic stroke: a meta-analysis of individual patient data from five randomised trials. Lancet 2016;387:1723-31.

3 Nogueira RG, Jadhav AP, Haussen DC, et al. Thrombectomy 6 to 24 hours after stroke with a mismatch between deficit and infarct. N Eng/ J Med 2018;378:11-21.

4 Albers GW, Marks MP, Kemp S, et al. Thrombectomy for stroke at 6 to 16 hours with selection by perfusion imaging. N Eng/ J Med 2018;378:708-18.

5 Alexandrov AV, Hall CE, Labiche LA, et al. Ischemic stunning of the brain: early recanalization without immediate clinical improvement in acute ischemic stroke. Stroke 2004;35:449-52.

6 Bang OY, Liebeskind DS, Saver JL, et al. Stunned brain syndrome: serial diffusion perfusion MRI of delayed recovery following revascularisation for acute ischaemic stroke. J Neurol Neurosurg Psychiatry 2011;82:27-32.

7 Kerr DM, Fulton RL, Lees KR, et al. Seven-day NIHSS is a sensitive outcome measure for exploratory clinical trials in acute stroke: evidence from the virtual international stroke trials archive. Stroke 2012:43:1401-3.

8 Rudilosso S, Urra X, Amaro S, et al. Timing and relevance of clinical improvement after mechanical thrombectomy in patients with acute ischemic stroke. Stroke 2019;50:1467-72.

9 Rangaraju S, Frankel M, Jovin TG. Prognostic value of the 24-hour neurological examination in anterior circulation ischemic stroke: a post hoc analysis of two randomized controlled stroke trials. Interv Neurol 2016;4:120-9.

10 Sajobi TT, Menon BK, Wang M, et al. Early trajectory of stroke severity predicts longterm functional outcomes in ischemic stroke subjects. Stroke 2017;48:105-10.

11 Mosimann PJ, Kaesmacher J, Gautschi D, et al. Predictors of unexpected early reocclusion after successful mechanical thrombectomy in acute ischemic stroke patients. Stroke 2018;49:2643-51.

12 Alexandrov AV, Grotta JC. Arterial reocclusion in stroke patients treated with intravenous tissue plasminogen activator. Neurology 2002;59:862-7.

13 Rapp Joseph H, Mang PX, Bo Y, et al. Cerebral ischemia and infarction from atheroemboli. Stroke 2003;34:1976-80.

14 Kloner RA, King KS, Harrington MG. No-reflow phenomenon in the heart and brain. Am J Physiol Heart Circ Physiol 2018;315:H550-62.

$15 \mathrm{Ng} \mathrm{FC}$, Coulton B, Chambers B, et al. Persistently elevated microvascular resistance postrecanalization. Stroke 2018;49:2512-5.

16 Gauberti M, Lapergue B, Martinez de Lizarrondo S, et al. Ischemia-reperfusion injury after endovascular thrombectomy for ischemic stroke. Stroke 2018;49:3071-4.

17 Direct evidence for calcium-induced ischemic and reperfusion injury. Available: https:// onlinelibrary.wiley.com/doi/abs/10.1002/ana.410260217 [Accessed 7 Jul 2019].

18 Nogueira RG, Gupta R, Jovin TG, et al. Predictors and clinical relevance of hemorrhagic transformation after endovascular therapy for anterior circulation large vessel occlusion strokes: a multicenter retrospective analysis of 1122 patients. J Neurointerv Surg 2015;7:16-21.

19 von Kummer R, Bourquain H, Bastianello S, et al. Early prediction of irreversible brain damage after ischemic stroke at CT. Radiology 2001;219:95-100.

20 Carmichael ST. Plasticity of cortical projections after stroke. Neuroscientist 2003:9:64-75

21 Hallett M. Functional reorganization after lesions of the human brain: studies with transcranial magnetic stimulation. Rev Neurol 2001;157:822-6.

22 Conti CR. The stunned and hibernating myocardium: a brief review. Clin Cardiol 1991;14:708-12.

23 Lancellotti P, Albert A, Berthe $C$, et al. Full recovery of contraction late after acute myocardial infarction: determinants and early predictors. Heart 2001;85:521-6. 